\title{
MERLIN (METHANE REMOTE SENSING LIDAR MISSION): AN OVERVIEW
}

\author{
C. Pierangelo ${ }^{* 1}$ B. Millet ${ }^{* 1}$, F. Esteve ${ }^{1}$, M. Alpers ${ }^{3}$, G. Ehret $^{4}$, P. Flamant ${ }^{5}$, S. Berthier ${ }^{5}$, F. Gibert ${ }^{5}$, \\ O. Chomette ${ }^{5}$, D. Edouart ${ }^{5}$, C. Deniel ${ }^{2}$, P. Bousquet ${ }^{6}$, F. Chevallier ${ }^{6}$ \\ ${ }^{1}$ Centre National d'Etudes Spatiales (CNES), 18 Av. E. Belin, 31401 Toulouse, FRANCE \\ *Email: clemence.pierangelo@cnes.fr,bruno.millet@cnes.fr \\ ${ }^{2}$ Centre National d'Etudes Spatiales (CNES), 2 place Maurice Quentin, 75039 Paris, FRANCE \\ ${ }^{3}$ Deutsches Zentrum für Luft-und Raumfahrt (DLR), Königswinterer Str. 522-524, 53227 Bonn, GERMANY \\ ${ }^{4}$ Deutsches Zentrum für Luft-und Raumfahrt (DLR), Münchener Straße 20, 82234 Weßling, GERMANY \\ ${ }^{5}$ Laboratoire de Météorologie Dynamique (LMD), Ecole Polytechnique, 91128 Palaiseau, FRANCE \\ ${ }^{6}$ Laboratoire des Sciences du Climat et de l'Environnement (LSCE) 91198 Gif-sur-Yvette, FRANCE
}

\begin{abstract}
The Methane Remote Sensing Lidar Mission (MERLIN), currently in phase $\mathrm{B}$, is a joint cooperation between France and Germany on the development, launch and operation of a methane ( $\mathrm{CH} 4)$ monitoring satellite. MERLIN is focused on global measurements of the spatial and temporal gradients of atmospheric $\mathrm{CH} 4$, the second most anthropogenic gas, with a precision and accuracy sufficient to constrain Methane fluxes significantly better than with the current observation network.

For the first time, measurements of atmospheric composition will be performed from space thanks to an IPDA (Integrated Path Differential Absorption) LIDAR (Light Detecting And Ranging). This payload is under the responsibility of the German space agency (DLR), while the platform (MYRIADE Evolutions product line) is developed by the French space agency (CNES). The IPDA technique relies on DIAL (Differential Absorption LIDAR) measurements using a pulsed laser emitting at two wavelengths, one wavelength accurately locked on a spectral feature of the methane absorption line, and the other wavelength free from absorption to be used as reference. This technique enables measurements in all seasons, at all latitudes. It also guarantees almost no contamination by aerosols or water vapour cross-sensitivity, and thus has the advantage of an extremely low level of systematic error on the dry-air column mixing ratio of $\mathrm{CH} 4$.
\end{abstract}

\section{INTRODUCTION}

Carbon dioxide $\left(\mathrm{CO}_{2}\right)$, methane $\left(\mathrm{CH}_{4}\right)$ and nitrous oxide $\left(\mathrm{N}_{2} \mathrm{O}\right)$ have been recognized by the International Panel of Climate Change (IPCC) [1] as the major greenhouse gases whose concentration has been directly modified by human activities.

The Methane Remote Sensing Lidar Mission (MERLIN), aims at global measurements of the spatial and temporal gradients of atmospheric $\mathrm{CH} 4$, the second most anthropogenic gas, with a precision and accuracy sufficient to constrain Methane fluxes significantly better than with the current observation network.

We show here an overview of the MERLIN mission: its science objectives, the measurement principle and the related expected performances, next the status of the project, including details on the space segment, the ground segment and the orbit.

\section{SCIENCE OBJECTIVES}

The major anthropogenic sources of $\mathrm{CH}_{4}$ are the emissions from energy production, landfills, waste treatment, cattle, rice fields, and incomplete biomass burning. In addition there are significant natural $\mathrm{CH}_{4}$ sources from wetlands. The observing Key regions are the Arctic regions (boreal forest, permafrost), Eurasia (anthropogenic emissions), the tropical regions (forest, wetlands).

For the regional quantification of greenhouse gas sources and sinks, the "top-down" approach relies on atmospheric gas concentration measurements and the use of an "inverted" model of atmospheric transport. At present the top-down approach is severely limited by the small number of observing stations. The goal of MERLIN is to improve this situation significantly by enhancing the $\mathrm{CH}_{4}$ observations to the point where regional to continental scale $\mathrm{CH}_{4}$ fluxes can be estimated to generally within less than $20 \%$ of their mean values. This requires high-precision and high accuracy observations, the ability to make measurements globally (even in the high-latitudes, during the winter months), and to avoid geographical biases.

To meet these objectives, MERLIN will provide column-weighted dry-air mixing ratios of $\mathrm{CH}_{4}$, referred to as $\mathrm{XCH}_{4}$, over the satellite sub-track. The IPDAlidar instrument considered for the MERLIN mission is not dependent on sunlight and will allow global coverage. Besides, the small footprint and the relative insensitivity to thin cirrus clouds and aerosol layers will allow observations in tropical regions and other 
areas that are frequently cloud covered or contaminated by biomass burning (e.g. boreal forests). Moreover, a considerable amount of $\mathrm{CH}_{4}$ is due to biomass burning of boreal forests which calls for a sensor that is immune to aerosol scattering. The MERLIN instrument shall operate in a range-gated mode and therefore will not be prone to biases that arise from aerosol scattering or scattering from thin cirrus layers.

\section{MEASUREMENT CONCEPT}

The core element of the MERLIN payload is the nadirviewing $\mathrm{CH}_{4}$ IPDA LIDAR system. This instrument measures the light scattered and reflected from the Earth's surface and cloud tops which are illuminated by laser pulses having slightly different wavelengths denoted as $\lambda_{\text {on }}$ and $\lambda_{\text {off }}$ as illustrated in Figure 1. The online wavelength $\lambda_{\text {on }}$ is accurately positioned in the trough of one of the $\mathrm{CH} 4$ absorption line manifold in the $1.64 \mu \mathrm{m}$ near-infrared spectral region. The measurement at $\lambda_{\text {off }}$ serves as the reference measurement with negligible absorption by the $\mathrm{CH} 4$ molecules in the path. The on- and off-line wavelengths are spectrally close enough to consider the atmospheric and surface interaction processes to be identical with the exception of $\mathrm{CH}_{4}$ absorption. Because the $\mathrm{CH}_{4}$ line is pressure broadened throughout the entire troposphere, a significant detuning of the online wavelength with respect to the $\mathrm{CH}_{4}$ absorption line center permits to put a higher weight on the contributions from the lower troposphere [2].

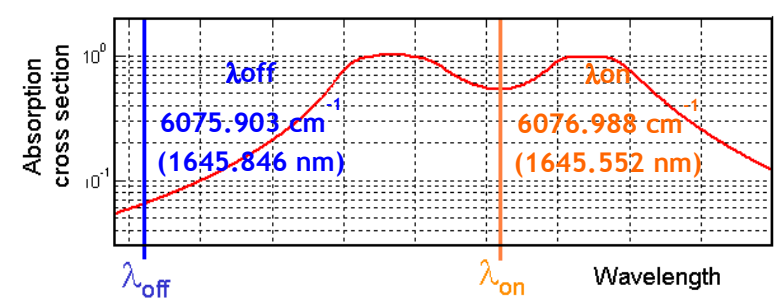

Figure 1: selected wavelength for MERLIN

The nadir viewing LIDAR instrument measures the reflected signals from ground along the track in a quasi-continuous operation mode. The distance on ground between successive on-/off-line laser pulses is about $350 \mathrm{~m}$ and depends on the pulse-pair repetition frequency (baseline: $20 \mathrm{~Hz}$ ).

Since the return signals are very weak, it is necessary to accumulate and average several single measurements of the return signals along the track in order to achieve the required measurement sensitivity for the $\mathrm{CH}_{4}$-column.

From the backscattered signals at both wavelengths, Pon, Poff,, a direct measurement of the differential absorption optical depth (DAOD) of methane is possible

$$
D A O D=\frac{1}{2} \ln \left(\frac{P_{\text {off }}}{P_{\text {on }}}\right)
$$

The DAOD is converted into vertically-integrated dry mixing ratio of methane, $\mathrm{XCH}_{4}$, which is the measurement used for assimilation in atmospheric transport models:

$$
\mathrm{XCH}_{4}=\frac{D A O D}{\int_{0}^{p_{\text {surf }}} \mathrm{WF}(p) d p}
$$

where the weighting function (WF) is integrated vertically from the surface pressure $\mathrm{P}_{\text {surf }}$ to the top-ofatmosphere.

It can be demonstrated that the weighting function, $\mathrm{WF}$, is:

$$
\mathrm{WF}=\frac{\left(\sigma_{o n}-\sigma_{o f f}\right)}{g\left(m_{d r y}+m_{H 2 O} x_{H 2 O}\right)}
$$

where $\sigma$ is the methane absorption cross-section for the "on" or "off" wavelength, m denotes the molecular mass of dry air or of water vapour, $g$ is the gravity acceleration, and $\mathrm{x}_{\mathrm{H} 2 \mathrm{O}}$ is the water vapour mixing ratio.

The cross-sections are computed with an absorption line model using spectroscopic parameters and information on meteorological profiles (pressure, temperature and humidity) The surface pressure $\mathbf{P}_{\text {surf }}$ in Eq. 2 is calculated using auxiliary data from NWP models (pressure and temperature) and information on the altitude of the scattering surface) where the lidar beam hits the ground.

\section{MISSION PRODUCTS}

The MERLIN mission will provide the following data products:

Level 0: for each individual laser shot the rangegated backscatter signal data together with auxiliary data such as ranging information, laser pulse energy, time, wavelength in a chronological data sequence. Level 1a: Level 0 data with all calibrations computed and appended.

- Level 1b: integrated signal at echo for off- and onwavelengths, slant-path DAOD, shot-by-shot and averaged, geo-location and time.

Level 2: column-weighted dry-air mixing ratios of methane (XCH4).

Level 3: visualizations of level 2 data such as XCH4 maps.

Aside from these data products, directly delivered by the MERLIN mission, level 4 data products are methane surface fluxes at various temporal and spatial scales, obtained through assimilation in transport model. Level 4 data products will not be part of the official MERLIN mission but provided by scientists at 
various scientific research centers using lower level MERLIN data.

In the context of the overall mission objective, additional data products can be derived from MERLIN. MERLIN secondary products can provide information about the surface (topography, estimates of the vegetation height and occasionally on the vertical structure, lidar retro-reflectance) and the atmosphere (cloud boundaries, including cloud base for small to moderate cloud optical thickness).

\section{EXPECTED PERFORMANCES}

MERLIN aims at providing global measurements of the spatial and temporal gradients of atmospheric methane $\left(\mathrm{CH}_{4}\right)$ with a precision and accuracy sufficient to significantly constrain Methane surface fluxes (Table 1). There are requirements on the random part of the error (high frequency, typically $>1 \mathrm{~Hz}$ ) and the systematic part (slowly varying component, e.g. orbital variations, or scene dependent errors). The very low level of systematic error aims at avoiding geographical biases in the $\mathrm{XCH} 4$ fields that could lead to uncertainties in fluxes.

Table 1: User Requirements on performances

\begin{tabular}{|c|c|c|c|}
\hline parameter & threshold & breakthrough & target \\
\hline $\begin{array}{l}\mathrm{XCH} 4 \text { random } \\
\text { error }\end{array}$ & $36 \mathrm{ppb}$ & $18 \mathrm{ppb}$ & $8 \mathrm{ppb}$ \\
\hline $\begin{array}{l}\mathrm{XCH} 4 \\
\text { systematic error }\end{array}$ & $3 \mathrm{ppb}$ & $2 \mathrm{ppb}$ & $1 \mathrm{ppb}$ \\
\hline Spatial coverage & Global & Global & Global \\
\hline Resolution & \multicolumn{3}{|c|}{ Horizontal: $50 \mathrm{~km}$, vertical: total column } \\
\hline
\end{tabular}

The main contributor for the random error on $\mathrm{XCH}_{4}$ is the error on the DAOD, due mostly to the Signal-toNoise Ratio of the instrument. In order to improve the random performance, averaging of the data of several shot pairs is foreseen, on distances of typically 10 to 50 $\mathrm{km}$.

Conversely to the random error, dominated by DAOD uncertainty, several elements almost equally contribute to the systematic errors: (i) the DAOD measured by the payload, (ii) the error on the surface pressure, caused by both the uncertainty on NWP products and the altitude (with platform and lidar ranging contributors), (iii) the error on the weighting function. Regarding this last contributor, the cross-section knowledge depends on external parameters (temperature profile from NWP, spectroscopic parameters), but also on the lidar itself, through the knowledge of the emitted frequency. As shown in Figure 1, the "on" frequency has been chosen in a local minimum of methane manifold in order to reduce the sensitivity to laser stability. Finally, processing itself may contribute to the systematic error through the introduction of scene-dependent biases, for example in the horizontal averaging step.

\section{STATUS OF THE PROJECT}

\subsection{SPACE SEGMENT}

The MERLIN satellite is based on a platform and a payload developed respectively by France and Germany.

The MERLIN platform, developed by Airbus Defence and Space-France under CNES contract, is based on the new MYRIADE product line so-named MYRIADE Evolutions. The MYRIADE Evolutions program offers: a new structure in order to reach $400 \mathrm{~kg}$ for satellite mass, an increase of the solar array capacity and the power distribution, a new adapted propulsion system, an improved Attitude and Orbital Control Systems (AOCS), an increase of the Payload data storage, a compatibility with low flight altitudes (Atomic Oxygen concerns).

Table 2. MERLIN Platform characteristics

\begin{tabular}{lll}
\hline \hline Satellite Mass & $\mathrm{kg}$ & 400 \\
\hline Platform Mass & $\mathrm{kg}$ & 260 \\
\hline Dimensions (body) & $\mathrm{mm}^{3}$ & $570 \times 940 \times 940$ \\
\hline Power consumption W & 180
\end{tabular}

The instrument is developed by Airbus Defence and Space-Germany under a DLR contract. Its main components are the instrument control unit, laser transmitter, a frequency reference unit, an energy calibration unit, receiver chain, receiving telescope and transmitter telescope. One of the main challenges of the MERLIN mission is to implement a complex LIDAR instrument on a small satellite platform with limited resources (power consumption, mass and volume allocations, thermal behavior, and radiation hardness). The main parameters of the MERLIN instrument can be found in table 3 .

Until now, no direct pumped laser at this wavelength is available, a laser concept based on a Nd:YAG pumped optical parametric oscillator (OPO) was selected. The laser will be operated in a pressurized housing. The stability of the laser and the actual knowledge of the laser frequency are crucial for this kind of instrument and therefore a compact and precise frequency reference is necessary: an etalon and a CCD-row for fringe measurements, combined with absolute wavelength calibration using a $\mathrm{CH}_{4}$ gas cell.

Aside from the laser, the receiver telescope is a challenging component of MERLIN. Its Field-of-View 
has to be large enough so that it "sees" all of the laser spot on ground.The other limiting factor is the size of the detector which should be small enough to limit the detector noise These factors finally result in a complex optical design. Furthermore, the thermal design of the telescope has to be in such a way that a purely passive thermal control system is sufficient. Due to the limited pulse energy and telescope size, the receiver and detector noise are both critical. More details on the instrument design and sub-components can be found in [3].

Table 3. MERLIN Payload characteristics

\begin{tabular}{lll}
\hline Payload Mass & $\mathrm{kg}$ & 119 \\
\hline Payload Volume & $\mathrm{mm}^{3}$ & $820 \times 830 \times 1010$ \\
\hline Payload Power & $\mathrm{W}$ & 150 \\
\hline On-line $\lambda \mathrm{on}$ & $\mathrm{nm}$ & 1645.552 \\
\hline Off-line $\lambda$ off & $\mathrm{nm}$ & 1645.846 \\
\hline Pulse energy & $\mathrm{mJ}$ & 9 \\
\hline Repetition rate & $\mathrm{Hz}$ & 20 (double pulse) \\
\hline Telescope diameter & $\mathrm{mm}$ & 690 \\
\hline \hline
\end{tabular}

\subsection{GROUND SEGMENT}

The MERLIN Command and Control Centre (CCC) is built from the future generic ISIS CCC (ISIS: Initiative for Space Innovation Standard). Its main tasks are to control and monitor the satellite and ensure its health over the mission lifetime. The CNES earth terminal network, based on stations implemented in Europe, Africa and America, will be used for commanding and receiving housekeeping data (S-band terminals), and for downloading of the Science data (X-band terminals).

The main functions of the payload ground segment are:

- Payload support functions: generation of commands to control the payload, monitoring of the payload housekeeping data for long term analysis, calibration and management of the payload onboard configuration.

- Payload data processing functionality for science data and products: data processing, archiving and dissemination up-to level 3.

\subsection{ORBIT DEFINITION}

For MERLIN, a near-polar sun-synchronous orbit (SSO) with an orbit height of about $500 \mathrm{~km}$, a Local Time Ascending Node (LTAN) of 06:00 or 18:00 (as shown in figure 9) and a repeat cycle of about 28 days is foreseen. The selection of the orbit is based on the following considerations:
- to get measurements on a global scale: selection of a near-polar sun-synchronous orbit

- the orbit should be as low as possible in order to get a return signal with an appropriate signal-to-noise ratio. The lower limit here is set by the platform (atomic oxygen and atmospheric drag).

- The LTAN selection is mainly based on satellite power issues.

- The repeat cycle determines the spacing between two subsequent revolutions and the re-visit time on certain areas on the globe. 28 day cycle leads to spacing between two subsequent tracks of about $100 \mathrm{~km}$ at the Equator.

Table 4. MERLIN orbit characteristics

\begin{tabular}{lcl}
\hline \hline Orbit type & & Sun-synchronous, dusk_dawn \\
\hline Semi major axis & $\mathrm{km}$ & $\approx 6884$ \\
\hline Altitude & $\mathrm{km}$ & $\approx 500$ \\
\hline Inclination & $\circ$ & $\approx 97.4$ \\
\hline Eccentricity & & $\approx 10^{-3}$ \\
\hline Repeat cycle & days & 28 \\
\hline \hline
\end{tabular}

\subsection{SCHEDULE}

The MERLIN project is currently in phase B: preliminary design. The MERLIN feasibility was demonstrated in the phase A, it was shown that it is possible to operate a complex IPDA LIDAR instrument on a MYRIADE Evolutions small satellite platform. The major milestones foreseen for the rest of the development are a Preliminary Design Reviews for satellite and payload in 2015, Critical Design Reviews in 2017 and a Launch date in December 2019.

\section{REFERENCES}

[1] Intergovernmental Panel on Climate Change, Climate Change 2007: The Physical Science Basis: Contribution of Working Group I to the Fourth Assessment Report of the IPCC, edited by S. Solomon et al., 996 pp., Cambridge Univ. Press, Cambridge, UK

[2] Kiemle, C., Quatrevalet, M., Ehret, G., Amediek, A., Fix, A., and Wirth, M.: Sensitivity studies for a space-based methane lidar mission, Atmos. Meas. Tech., 4, 2195-2211, doi:10.5194/amt-42195-2011, 2011.

[3] C. Stephan, et al., 2011: MERLIN - a Space-based Methane Monitor, Proceedings of the SPIE, Volume 8159, 815908-15. 